\title{
A Scalable K-hop Clustering Algorithm for Pseudolinear MANET
}

\author{
Tanjil Ahmed \\ Stamford University Bangladesh \\ Dhaka, Bangladesh
}

\author{
Ambreen Zaman \\ University of Bremen \\ TZI, Bremen, Germany
}

\author{
Md. Abdur Rahman \\ Centre for Advanced Research in Sciences \\ University of Dhaka, Bangladesh
}

\author{
Mahfida Amjad \\ Stamford University Bangladesh \\ Dhaka, Bangladesh
}

\begin{abstract}
The scalability issue is essential to gain commercial success in MANET. However, there are some existing constraints such as low bandwidth, node mobility, energy consumption, and the broadcast nature of wireless communication that make the network complex to maintain. Furthermore, as the network size increases, communication costs may consume a large proportion of the bandwidth. Other parameters such as node mobility, node density, and traffic load can also impair network scalability. In this research paper, we proposed a new algorithm, K-hop clustering scheme for Pseudolinear MANET (KHPM) where cluster topology remains stable in terms of Khop and more dense network. The strategy used stability metric and Doppler Value (DV) between nodes, which exchanged packets obtained by Doppler shift. A node's smaller DV indicates lower mobility with higher stability. The proposed technique has been analysis hypothetically, which shows that the proposed algorithm is able to consider mobility as a key feature compared to existing SKCA method. In addition, the number of hops of a cluster is twice in KHPM compared to existing THPM technique.
\end{abstract}

\section{General Terms}

Ad hoc wireless network, Clustering.

\section{Keywords}

K-hop clustering, Cluster-head, Cluster-member, Pseudolinear Mobile Ad Hoc Network, Doppler Value, Network scalability, Cluster stability, Cluster based routing.

\section{INTRODUCTION}

Mobile Ad Hoc network (MANET) is self configured and less infrastructure network of mobile devices [1]. The MANET consists of autonomous mobile nodes, organized themselves without any aid of administration and topology is changed dynamically. If the network has a flat topology then the size of routing table will be proportional to the number of nodes in the entire network. However, the degree of network also conflict with scalability in flat structure [2]. Because of flat topology MANET encounters several constraints like low bandwidth, node with mobility, low energy, and the broadcast nature of wireless communication [2]. In addition, as the network size increases, communication costs may consume large proportion of the bandwidth [2].

Hierarchical routing is a remarkable solution for building a scalable network. One promising approach to build hierarchy among the nodes is clustering [14]. The number of hops of the mobile nodes is an important issue in cluster based MANET.
Most researches have focused on single hop clustering, which allows simple local management within each cluster [3][4] [5][6][7]. MOBIC [4] is a one hop clustering algorithm which tightens the connection between nodes for minimizing the influence of mobile nodes' movement. Performance of this scheme may be degraded if mobile nodes move randomly.

There are several multi-hops clustering schemes have been designed for MANET [8], [9], [10]. However, there is no such remarkable clustering scheme proposed for Pseudolinear Mobile Ad hoc Network in which scalability considered .The nodes of the Pseudolinear Mobile Ad hoc Network move in a nearly straight line beyond random displacement in respect of direction and motion, for example, aircraft, ships, trains, and cars on highways, these nodes are known as pseudolinear mobile entity, and the communication among the pseudolinear mobile entities is known as Pseudolinear Highly Mobile Ad hoc Network (PHMANET) [11]. It focuses on a clustering technique for the nodes with slow changes of node's moving direction and speed. If mobile nodes change its mobility pattern frequently, the performance of this clustering scheme may degrade. This clustering technique considers single hop, which causes frequently change of cluster-heads and the diameter of each cluster is also small.

To increase hop, Two Hop clustering scheme for Pseudolinear MANET (THPM) [12] proposed a two hop clustering scheme for PHMANET [11] which is the refinement of [13]. It uses stability metric and Doppler Value (DV) for cluster formation. THPM develops an efficient equation for calculating two hop neighbors to forming a two hop cluster in the network. For achieving the scalability issue in case of large network it is needed to consider K-hop clustering. There is a K-hop clustering schemes for MANET like SKCA [18], however there is no such clustering scheme for PHMANET considering K-hop.

To form a scalable and stable cluster network this paper proposed a Scalable K-hop Clustering Algorithm for Pseudolinear MANET (KHPM) scheme for PHMANET. The proposed KHPM technique is the extension of THPM [12]. KHPM develops an equation for calculating K-hop neighbors for forming a K-hop cluster in the network. It develops an equation based on Euclidean distance by ignoring direct link between two nodes and excluding the self node. It also proposes an algorithm for K-hop cluster maintenance taking the help of SKCA [18].

The proposed technique has been analysis hypothetically, which shows that the new scalable clustering algorithm (KHPM) is able to consider mobility as a key feature 
compared to existing SKCA method. In addition, the number of hops of a cluster is twice in KHPM compared to THPM.

The rest of this paper is organized as follows. Section 2 describes the overview of some previous clustering algorithms. Section 3 and 4 illustrated the proposed methodology and algorithm evaluation respectively. Finally section 5 concludes this paper with future research direction.

\section{LITERATURE REVIEW}

Clustering is an important research topic for Mobile Ad hoc Networks (MANETs) because clustering makes it possible to guarantee basic levels of system performance, such as throughput and delay, in the presence of both mobility and a large number of mobile terminals [2]. A variety of different approaches have been proposed for one hop ad hoc clustering scheme which typically focus on different performance metrics such as low maintenance clustering, which provides a stable cluster infrastructure by minimizing clustering-related maintenance cost, especially reducing re-clustering situations. Least Cluster Change (LCC) [15], 3-hop Between Adjacent Cluster-heads (3hBAC) [16], Lin's Algorithm [3], and Passive Clustering (PC) [17] are some of the examples of lowmaintenance clustering scheme.

LCC [15] a one hop clustering scheme which limits reclustering situations and reduces clustering control overhead. $3 \mathrm{hBAC}$ [16] eliminating ripple effect of re-clustering; producing non-overlapping cluster structure and eliminating unnecessary small clusters. Lin's algorithm [3] limits reclustering situations and reducing clustering control overhead; eliminating cluster-heads in cluster maintenance to avoid overloaded nodes. PC [17] is another one hop clustering algorithm which eliminates explicit control message for clustering and form and maintain a cluster structure only when some mobile nodes have packets to send; suppress the number of gateways to achieve flooding efficiency.

On the other hand, mobility-aware clustering utilizes mobile nodes' mobility behavior for cluster construction and maintenance and assigning mobile nodes with low relative speed to the same cluster to tighten the connection in cluster. Schemes are Mobility Based Metric for Clustering (MOBIC) [4] and Distributed Dynamic Clustering Algorithm (DDCA) [8].

MOBIC [4] is a one hop clustering algorithm that minimizes the influence of mobile nodes' movement on cluster topology updates in terms of re-affiliation and re-clustering based on mobile nodes' explicit relative speed; tightening the connection between mobile nodes residing in the same clusters.

DDCA [8] is a multi-hop clustering algorithm. It reduces the influence of mobile nodes' movement on cluster topology updates in terms of re-affiliation and re-clustering, adjusting the dominant routing mechanisms based on a network's mobility behavior. It works than MOBIC in a dynamic environment where mobile nodes are continuously moving.

To enhance the stability of the cluster topology and reduce the clustering overhead SKCA (Stable K-hop Clustering Algorithm for Routing in Mobile Ad Hoc Networks) [18] is a clustering algorithm which introduces a two-round clusterhead election that reduces the diffusion of the cluster information in the K-hop neighborhood. This algorithm attempts to enhance the stability of the cluster topology and reduce the clustering overhead. SKCA proposes a new maintenance function that allows two cluster-heads exists in the K-hop neighborhood for particular situations.
PHMANET [11], it can be seen that the proposed schemes can form stable clusters containing members that remain within their associated clusters for a longer period of time, despite the targeted system having node speeds exceeding normal MANET scenarios. In [11], stability is defined in terms of cluster membership. Although the proposed clustering algorithm [11] ensures that no cluster-heads are within the range of each other in the initial cluster formation, prevent and thus may cause cluster contentions. It introduces two new stability driven clustering algorithms, one is Dubbed dynamic Doppler Velocity Clustering (DDVC) and another is Dynamic Link Duration Clustering (DLDC). The aim of the proposed clustering algorithms is to form stable clusters considering the mobility and position parameters of the nodes. The algorithms presented in [11] are suited for the possibility of reliable data sharing and communication between highly mobile vehicles, depending on the amount of reliable mobility information available in the targeted system.

THPM [12] is the refinement of [13] and extension of PHMANET [11]. THPM offers clustering algorithm for two hop neighbor for PHMANET. It uses node mobility in terms of the average cluster-head lifetime of cluster-members. Here, the cluster-heads are selected based on node stability, which has been calculated using Doppler value.

The analysis of existing clustering approaches shows that different clustering algorithms have been proposed for MANET such as low-maintenance, mobility-aware, one hop, multi hop, stable K-hop etc. However, there is no such clustering scheme to solve PHMANET scalability problem considering K-hop neighbor for large network.

\section{PROPOSED KHPM METHODOLOGY}

Building clusters in a network is one of the prominent approaches to solve the scalability problem in MANET. After building hierarchical structure of the mobile nodes, it is important to examine cluster structure stability, control overhead of cluster, energy consumption of mobile nodes to ensure the network stability as well as scalability. The proposed Scalable K-hop Clustering Algorithm for Pseudolinear MANET (KHPM) method has been proposed to overcome the scalability and building hierarchical structure limitations. The KHPM is a new algorithm to provide scalable and stable network considering the total number of mobile nodes in the network. The strategy used stability metric and Doppler Value (DV) between nodes, which exchanged packets obtained by Doppler shift. A node consist of smaller DV, that means lower mobility provide higher stability. The proposed method is divided into following four steps.

\section{Step 1: Calculate Doppler Shift}

\section{Step 2: K-Hop Neighbor Calculation}

\section{Step 3: Cluster Formation}

\section{Step 4: Cluster Maintenance}

Detail descriptions of the above steps are given in the following subsections. 


\subsection{Calculate Doppler Shift}

Doppler Value (DV) is measured based on the relative velocity between nodes obtained from the Doppler shift of control packets exchanged between nodes during the clustering process. The smaller DV of a node means lower mobility and higher stability than its neighbors and this node has the quality of being the cluster-head in KHPM.

During clustering process 'Hello' message is exchanged to the neighboring nodes indicating its existence in the network. The DV of the Hello messages of each node is calculated by first measuring the Doppler shift of the Hello messages. Doppler shift of the Hello message determines the nodes to be used in cluster as a head-node or a member-node. In the network, it is assumed that all the participating nodes initially remains in Null and can be used as either cluster-head or cluster-member.

The Doppler shift of two mobile nodes is related to the relative velocity, which is calculated as follows [12].

$V=C\left[\left(\frac{F}{F 0}\right)-1\right]$

Where:

$V=$ Relative velocity between to communicating nodes

$C=$ Speed of light

$F=$ Expected frequency of the signal/packet and

$F O=$ Observed frequency of the signal/packet.

In case of approaching and receding nodes, DV is calculated using relative velocity of two nodes taking into account the effect of approaching and receding node. The following equation is used to measure DV for approaching nodes.

$$
\text { If }\left(\frac{F}{F 0}\right)<1 \text { then } D V=C|V|
$$

For approaching nodes, the observed frequency turns out to be smaller than the expected frequency.

For receding nodes:

$$
\text { If }\left(\frac{F}{F 0}\right)>1 \text { then } D V=2 C|V|
$$

For receding nodes, however, the observed frequency is greater than the expected frequency. Receding nodes are considered half as stable as approaching nodes, as nodes that are approaching are generally be within communication range of each other for twice as long as nodes that are receding from each other

The DVs of all the received Hello messages are stored at each node. These are then used to calculate the Sum of the DVs (DVS). The DVS reflects the relative stability of the node with respect to its neighbors as it defines the relative mobility based on the DV of the nodes. A smaller DVS will result in lower mobility and, hence, higher stability of a node with respect to its neighbors.

$\mathrm{K}$-hop neighbor calculation is discussed in details in the next step.

\subsection{K hop Neighbor Calculation}

This research is proposing a clustering algorithm KHPM for $\mathrm{K}$-hop neighboring information in a network. It uses K-hop neighboring information for forming a cluster. For calculating the K-hop neighbor, this research uses the concept from [12] which is a solution of topology control based on two hop neighbors' information for PHMANET [11]. The mechanism for calculating the K-hop neighbor has been described below.

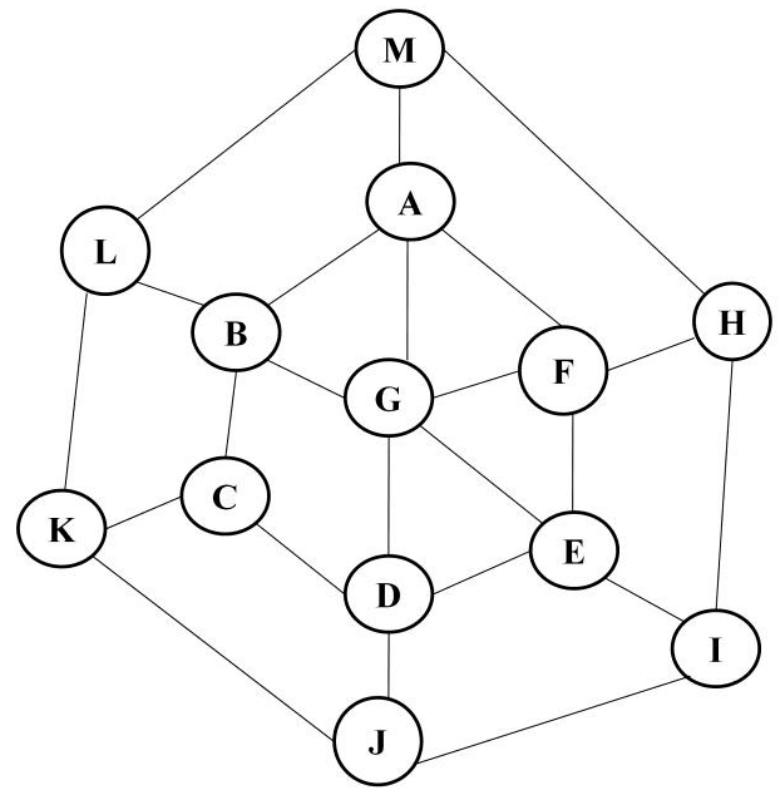

Fig. 1: Example of K-Hop cluster

Let each node in Fig. 1 knows its K-hop neighbor information (based on Euclidean distance [12]). Then the one hop neighbor $(\mathrm{OHN})$ lists of each node are:

$$
\begin{aligned}
& \text { OHN }(A)=\{B, F, G, M\} \\
& \text { OHN }(B)=\{A, C, L, G\} \\
& \text { OHN }(C)=\{D, B, K\} \\
& \text { OHN }(D)=\{C, E, J, G\} \\
& \text { OHN }(E)=\{F, D, G, I\} \\
& \text { OHN }(F)=\{A, E, G, H\} \\
& \text { OHN }(G)=\{B, A, F, G, D\} \\
& \text { OHN }(H)=\{I, M, F\} \\
& \text { OHN }(I)=\{H, J, E\} \\
& \text { OHN }(J)=\{D, I, K\} \\
& \text { OHN }(K)=\{J, L, C\} \\
& \text { OHN }(L)=\{K, M, B\} \\
& \text { OHN }(M)=\{A, H, L\}
\end{aligned}
$$

Now K-hop neighbor calculation of node A will be:

$$
\begin{aligned}
& F H N(A)=[O H N(B)-O H N(A)] U[O H N(F)-O H N(A)] \\
& U[O H N(G)-O H N(A)] U[O H N(M)-O H N(A)] \\
& =[\{A, C, L, G\}-\{B, F, G, M\}] \\
& U[\{A, E, G, H\}-\{B, F, G, M\}] \\
& U[\{B, A, F, G, D\}-\{B, F, G, M\}] \\
& U[\{A, H, L\}-\{B, F, G, M\}] \\
& =\{A, C, L\} U\{A, E, H\} U\{A, D\} U\{A, H, L\} \\
& F_{H N_{(A)}}=\{A, C, D, H, E, L\}
\end{aligned}
$$

Where, FHN denotes Four Hop Network. 
By excluding self node, FHN $(A)=\{C, D, H, E, L\}$

In the same way, K-hop neighbor calculation for node $\mathrm{E}$ will be as below.

$F H N(E)=[O H N(F)-O H N(E)] U[O H N(D)-O H N$

$(G)] U[O H N(G) O H N(E)] U[O H N(I) O H N(E)]$

$$
=[\{A, E, F, G\}-\{F, D, G, I\}] U[\{C, E, J, G\}-\{F, D,
$$

$G, I\}] U[\{B, A, F, G, D\}-\{F, D, G, I\}]$

$$
\begin{aligned}
& =[\{H, I, F\}-\{F, D, G, I\}] \\
& =[\{A, E, H\} U\{C, E, J\} U\{B, A\} U\{H, J, E\}]
\end{aligned}
$$$$
F H N_{(E)}=\{A, B, C, E, H, J\}
$$

By excluding self node and ignoring the direct link between the nodes:

$$
F H N_{(E)}=\{A, B, C, H, J\}
$$

Therefore, K-hop neighbor calculation general equation can be written as below.

Let consider set of nodes:

$$
\begin{aligned}
& U=U_{1}, U_{2}, U_{3}, \ldots \ldots U_{n} \\
& \text { OHN }_{U n} \text { list }=V_{1}, V_{2}, V_{3}, \ldots \ldots \ldots V_{n}
\end{aligned}
$$

\begin{tabular}{|c|c|}
\hline \multicolumn{2}{|c|}{ Algorithm 1: Cluster formation for K-hop neighbor } \\
\hline \multicolumn{2}{|c|}{ Initialization: } \\
\hline State: & Null \\
\hline Node identifier: & node $I d$ \\
\hline One hop neighbor : & $\mathrm{OHN}$ \\
\hline K-hop (four hop) neighbor: & FHN \\
\hline Set of node $(\mathrm{V})$ : & $\mathrm{U}_{1}, \mathrm{U}_{2}, \mathrm{U}_{3} \ldots \ldots \ldots \mathrm{Un}_{\mathrm{n}}$ \\
\hline$\left(\mathrm{U}_{\mathrm{n}}\right)$ list : & $\mathrm{V}_{1}, \mathrm{~V}_{2}, \mathrm{~V}_{3} \ldots \ldots \ldots \mathrm{V}_{\mathrm{n}}$ \\
\hline Sum of Doppler values: & DVS \\
\hline Own DVS : & O_DVS \\
\hline Receiving DVS: & R_DVS \\
\hline Message type (packet) : & HELLO, DVS \\
\hline
\end{tabular}

Then,

$$
\begin{gathered}
\mathrm{FHN}_{\mathrm{Un}}=\left[\mathrm{OHN}_{\mathrm{V} 1}-\mathrm{OHN}_{\mathrm{Un}}\right] \mathrm{U}\left[\mathrm{OHN}_{\mathrm{V} 2}-\mathrm{OHN}_{\mathrm{Un}}\right] \\
\mathrm{U}\left[\mathrm{OHN}_{\mathrm{V} 3}-\mathrm{OHN}_{\mathrm{Un}}\right] \mathrm{U}\left[\mathrm{OHN}_{\mathrm{Vn}}-\mathrm{OHN}_{\mathrm{Un}}\right]
\end{gathered}
$$

Where, $\mathrm{U}_{\mathrm{n}} \neq \mathrm{V}_{\mathrm{n}}$ and there is no direct link between $\mathrm{U}_{\mathrm{n}}$ and $\mathrm{V}_{\mathrm{n}}$.

\section{Round 1}

1: Begin

2: [each node]

Hello message (node ID, NULL) to its FHNS using [5]

$\mathrm{FHN}_{(\mathrm{Un})}:\left[\mathrm{OHN}_{(\mathrm{Un})}-\mathrm{OHN}_{(\mathrm{Un})}\right] \mathrm{U}\left[\mathrm{OHN}_{(\mathrm{V} 2)}-\mathrm{OHN}_{(\mathrm{Un})}\right]$ $\mathrm{U}\left[\mathrm{OHN}_{(\mathrm{V} 3)}-\mathrm{OHN}_{(\mathrm{Un})}\right] \ldots\left[\mathrm{OHN}(\mathrm{Un})-\mathrm{OHN}_{(\mathrm{Un})}\right]$

3: role of the node jump [1. pre-cluster head 2. premember]

4: Jump 1 [if highest number of null neighbor]; Otherwise jump 2

5: Broadcast HELLO [node declares its role]

6: [ Hello receiving node ] calculate DVS of all HELLO Broadcast DVS (node ID, DV_S) to their FHN $\mathrm{F}_{\mathrm{s}}$

\section{Round 2}

1: Role: Pre-cluster head [all Pre-cluster head elected after first round]

2: [DVS receiving nodes] compare R_DVS with O_DVS If O_DVS < R_DVS

3: Broadcast cluster head claim (cluster ID, O_DVS) to their FHNs containing smallest O_DVS node will be cluster-head.

4: [Cluster-head claim receiving nodes] can wish to join the cluster.

Send join request (cluster ID, node ID)

5: [cluster-head]

Send join accept (cluster ID, node Id)

6: [request accepted nodes]

Join the cluster and periodically broadcast, Clustermember (cluster ID, node ID)

7: $\quad$ End

Round 1: HELLO message broadcast by each node to their FHNs indicating its existence in the network in Step 2. In Step 3 role of node jump to the pre cluster-head or pre member. After first round a node can be a potential cluster head if it has highest number of null neighbor. Otherwise, it has becomes a potential member in Step 4. Hello receiving nodes calculate the DVS and broadcast to its FHNs in Step 5 and 6.

Round 2: In Step 1 all pre cluster-head elected after the first round participated to find the effective cluster-head. Compare the receiving DVS with its own DVS (O_DVS). In the K-hop cluster, if any node has O_DVS < R_DVS, it broadcast cluster-head claim (cluster-ID; O_DVS) to its FHN. Smallest O_DVS node will be the cluster-head in Step 2. Here cluster ID is equal to the ID of the cluster-head claim node. A node may to join the cluster when cluster-head claim packet. A node must send a join request message (cluster ID, node ID) if it wishes to join in a cluster. This request message accepted by cluster-head and sends a join accept (cluster ID, node ID) message to the requesting nodes in Step 3 and 4. Periodically broadcast cluster-member (cluster ID, node ID) notifying its role by the cluster-head to accept nodes and its associated cluster of its existence in Step 5 and 6.

This proposed algorithm for clustering scheme operates in two phases, basic cluster formation and cluster maintenance which have been explained on the following sections.

\subsection{Basic Cluster Formation}

To select cluster-head, each node collects the required information to decide its role in the topology of cluster. Two round selection of cluster-head based on node stability which has been calculated using Doppler value makes KHPM more scalable. In KHPM, role of the node in a cluster topology could be modeled as role changes diagram on the basis of [18]. Fig. 2 exhibit 6 role changes of one node are as follows. Each node can be in one of the following role.

NULL: In any cluster it is the basic role of all node means that its roles are yet to be defined. When a node in Null role it become the new member of cluster or left from cluster. That means it is the initial role for all nodes.

Pre-member: It denotes that, in first round the node has been defeated and it becomes a cluster-member node. Pre-cluster heads jump to pre-member role if a node among to its one hops neighbor or several. 


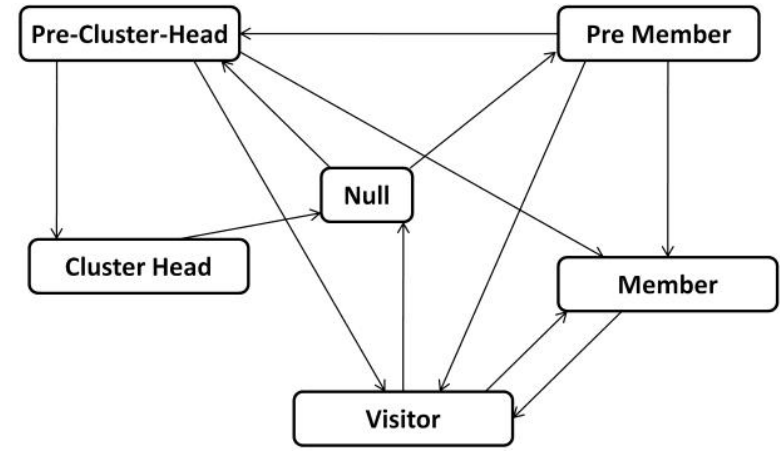

Fig. 2: Role of the node in a cluster in KHPM.

Cluster-head: This node denotes that the node creates a new cluster and it becomes the leader of this cluster. Inside a cluster, cluster-head is responsible for collecting information from member.

Cluster-member: A cluster-member is usually called an ordinary node which has joined a cluster-head and belongs to its cluster.

Visitor member: To reduce neighborhood the node invites itself to join a cluster. When it cannot find any cluster-head this role introduces and find at least one direct neighbor which is in a cluster member role.

Continuous Cluster Maintenance: In ad hoc network on cluster topology changes i.e. when node already in a cluster and wants to leave the cluster for example: changes are, cluster-head role renouncement, role changes, new cluster creation. For reducing the changes of cluster topology and increase cluster lifetime KHPM has an effective mechanism for node activation and deactivation. If a node is connected or disconnected from its neighboring nodes incidence, the performance of network may degrade by the frequent change of cluster and also consumes bandwidth.

\subsection{Cluster Maintenance}

The cluster maintenance mechanism can be performed in two cases: one is packet receiving incident and another is node leaving incident.

Packet Receiving Incidence: The fundamental goal of node is to join a cluster. In every mobile network, when a node wish to join in a cluster it monitors its neighbor node through HELLO packet. In that time two cases occurs:

Case 1: A non member node wants to join in a cluster, when it comes to close to its cluster or switched on its power inside the cluster.

Case 2: When a non member node within K-hop range cluster, the node tires to join on receiving HELLO packet.

Node Leaving Incidence: Within a cluster, when a member node does not receive periodic broadcast from a cluster-head then the following two cases occur:

Case 1: When a member node does not receive periodic broadcast from a cluster head:

- The node leaves from its cluster or switched off its power at that time. Then cluster-head removes this member node from its list.

Case 2: A node loses the path for connecting the original cluster and the node is not part of any cluster.
- Then the distance between the cluster-head is for enough to join the cluster. In this time this node begins broadcast periodic join request message to find a new cluster to join.

- A new cluster-head selects by the node which is able to connect the K-hop cluster-member in this situation.

Then all nodes again go back to Null state and perform the process basic cluster formation as same way.

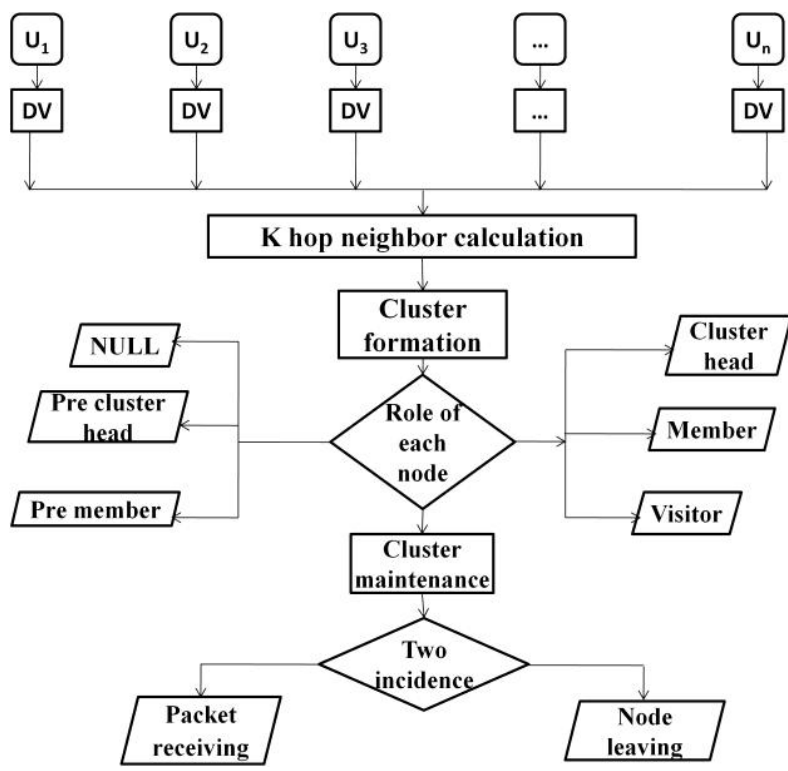

Fig. 3: Overview of the KHPM Method.

\subsection{Summary of Methodology}

The proposed scalable clustering algorithm is implemented to develop a K-hop neighbor calculation equation to forming a $\mathrm{K}$-hop cluster in the network. It develops the equation based on Euclidean distance by ignoring direct link between two nodes and excluding the self node. The strategy used stability metric and Doppler Value (DV) between nodes obtained by Doppler shift. A node consists of smaller DV that means lower mobility and provides higher stability.

\section{HYPOTHETICAL ANALYSIS}

In this section hypothetical analysis has been presented to provide some logical aspect comparing proposed algorithm KHPM with existing algorithm THPM along with SKCA. Effectiveness of KHPM is measured based on two main logical statements which are structural differences of topology and differences of performance characteristic.

Table 1. Structural differences of topology

\begin{tabular}{|l|l|l|}
\hline Key Point & KHPM & SKCA \\
\hline $\begin{array}{l}\text { Network } \\
\text { Type }\end{array}$ & $\begin{array}{l}\text { KHPM proposed } \\
\text { for Pseudolinear } \\
\text { MANET }\end{array}$ & $\begin{array}{l}\text { SKCA proposed } \\
\text { for wireless } \\
\text { MANET }\end{array}$ \\
\hline $\begin{array}{l}\text { Cluster- } \\
\text { head }\end{array}$ & $\begin{array}{l}\text { Cluster-head usiong } \\
\text { selection by using } \\
\text { Doppler Value } \\
\text { (DV) }\end{array}$ & $\begin{array}{l}\text { Cluster-head } \\
\text { selection based on } \\
\text { node connectivity }\end{array}$ \\
\hline
\end{tabular}


The whole analysis of logical result is shown in Table 1 and Table 2. Structural differences of topology comparison between the KHPM and SKCA have been demonstrated in Table 1 considering network type and cluster-head selection parameters.

Table 2. Differences of performance characteristics

\begin{tabular}{|l|l|l|}
\hline Characteristic & KHPM & SKCA \\
\hline Mobility & $\begin{array}{l}\text { Used DV for } \\
\text { calculating } \\
\text { mobility }\end{array}$ & $\begin{array}{l}\text { Does not provide } \\
\text { mobility }\end{array}$ \\
\hline $\begin{array}{l}\text { Energy } \\
\text { Consumption }\end{array}$ & $\begin{array}{l}\text { Maintains energy } \\
\text { consumption } \\
\text { level }\end{array}$ & $\begin{array}{l}\text { No indication of } \\
\text { energy } \\
\text { consumption }\end{array}$ \\
\hline
\end{tabular}

The performance between KHPM and SKCA methods based on mobility and energy has been shown in Table 2. According to the Table 2, mobility is calculated in KHPM using DV where mobility is not considered in SKCA. In addition, the proposed system maintains nodes energy consumption however; existing system has no indication about it.

\section{DISCUSSION}

This research introduces KHPM, a new clustering algorithm for Pseudolinear MANET. In this investigation, the proposed KHPM is compared with SKCA on the basis of topological differences i.e. network type and cluster head selection process. KHPM proposed for PHMANET, which is one of the most prominent clustering techniques in MANET. The initial objective of this work is to develop a clustering technique that can ensure the longevity of cluster to manage K-hop ad hoc network in an efficient and scalable way. The second aim is to define efficient cluster-head selection process. In KHPM, cluster-head is selected by using Doppler value (DV), where as SKCA selects cluster-head based on node connectivity which is inconvenient for large scale network.

Table 2 shows performance characteristic based on mobility and energy where KHPM provides mobility but existing SKCA does not. Moreover, KHPM maintain energy consumption in marginal condition which make it more convenient.

In Table 3 performance comparison has been demonstrated in terms of number of hops supported by proposed algorithm and minimum number of nodes in a cluster between KHPM and existing THPM methods.

Table 3. Number of Hops and Nodes Comparison

\begin{tabular}{|c|c|c|}
\hline Method & $\begin{array}{c}\text { Number of } \\
\text { Hops }\end{array}$ & $\begin{array}{c}\text { Minimum Number of } \\
\text { nodes in a cluster }\end{array}$ \\
\hline KHPM & 4 & $12+1$ (cluster-head) \\
\hline THPM & 2 & $6+1$ (cluster-head) \\
\hline
\end{tabular}

The number of hops of a cluster is twice in KHPM compared to THPM. In addition, the minimum number of nodes for a cluster is double (excluding the cluster-head) in KHPM compared to THPM. Therefore, the proposed KHPM technique can support more nodes along with increasing number of hops which is scalable in terms of $\mathrm{K}$ hop clustering network.

\section{CONCLUSION}

This paper presented a new efficient and scalable K-hop clustering algorithm for Pseudolinear MANET. Where, a cluster topology remains stable in terms of K-hop and increasing number of nodes in the network. The proposed scalable clustering approach develops an equation based on Euclidean distance by ignoring direct link between two nodes and excluding the self node. The strategy used stability metric and Doppler Value (DV) between nodes, which exchanged packets obtained by Doppler shift. A node's smaller DV indicates lower mobility and higher stability. The proposed technique has been described hypothetically, which shows that the new scalable clustering algorithm provides node mobility but existing SKCA does not. In addition, the number of hops of a cluster is twice in KHPM compared to existing THPM technique. However, this research is limited to algorithm analysis hypothetically and should be applied with simulation. Evaluating this algorithm with real complexities is the future direction of work.

\section{REFERENCES}

[1] Loo, Jonathan, Jaime Lloret Mauri, and Jesus Hamilton Ortiz, eds. Mobile ad hoc networks: current status and future trends. CRC Press, 2016.

[2] Jane Yang Yu and Peter Han Joo Chong. A survey of clustering schemes for mobile ad hoc networks. IEEE Communications Surveys \& Tutorials, 7(1):32-48, 2005.

[3] Chunhung Richard Lin and Mario Gerla. Adaptive clustering for mobile wireless networks. IEEE Journal on Selected areas in Communications, 15(7):1265-1275, 1997.

[4] Prithwish Basu, Naved Khan, and Thomas DC Little. A mobility based metric for clustering in mobile ad hoc networks. In Distributed computing systems workshop, 2001 international conference on, pages 413-418. IEEE, 2001.

[5] Kaixin $\mathrm{Xu}$ and Mario Gerla. A heterogeneous routing protocol based on a new stable clustering scheme. In MILCOM 2002. Proceedings, volume 2, pages 838-843. IEEE, 2002.

[6] Yanlei Shang and Shiduan Cheng. A stable clustering formation in mobile ad hoc network. In Wireless Communications, Networking and Mobile Computing, 2005. Proceedings. 2005 International Conference on, volume 2, pages 714-718. IEEE, 2005.

[7] Aravindhan Venkateswaran, Venkatesh Sarangan, Natarajan Gautam, and Raj Acharya. Impact of mobility prediction on the temporal stability of manet clustering algorithms. In Proceedings of the 2nd ACM international workshop on Performance evaluation of wireless ad hoc, sensor, and ubiquitous networks, pages 144-151. ACM, 2005.

[8] A Bruce McDonald and Taieb F Znati. Design and performance of a distributed dynamic clustering algorithm for adhoc networks. In Simulation Symposium, 2001. Proceedings. 34th Annual, pages 27 35. IEEE, 2001.

[9] Tomoyuki Ohta, Shinji Inoue, and Yoshiaki Kakuda. An adaptive multihop clustering scheme for highly mobile 
ad hoc networks. In Autonomous Decentralized Systems, 2003. ISADS 2003. The Sixth International Symposium on, pages 293-300. IEEE, 2003.

[10] Alan D Amis and Ravi Prakash. Load-balancing clusters in wireless ad hoc networks. In Application-Specific Systems and Software Engineering Technology, 2000. Proceedings. 3rd IEEE Symposium on, pages 25-32. IEEE, 2000.

[11] Ehssan Sakhaee and Abbas Jamalipour. Stable clustering and communications in pseudolinear highly mobile adhoc networks. IEEE Transactions on Vehicular Technology, 57(6):3769-3777, 2008.

[12] Ambreen Zaman, Mahfida Amjad, and Kazi Sakib. Two hop clustering scheme for pseudolinear mobile ad hoc network (thpm). Asian Journal of Information Technology, 11(6):261-269, 2012.

[13] Mahfida Amjad, Ambreen Zaman, and Kazi Sakib. Efficient Scalable Clustering Scheme for Pseudolinear Mobile Ad hoc Network (THPM), The 7" Int'l Conference on Wireless Communications, Networking and Mobile Computing (WiCOM), Sep 23-25, 2011, Wuhan,China
[14] Yang Xiao, Jie Li, and Yi Pan. Ad-hoc and sensor networks: Wireless networks and mobile computing (wireless networks and mobile computing, v. 2). 2005.

[15] Ching-Chuan Chiang, Hsiao-Kuang Wu, Winston Liu, and Mario Gerla. Routing in clustered multihop, mobile wireless networks with fading channel. In proceedings of IEEE SICON, volume 97, pages 197-211, 1997.

[16] JY Yu and Peter HJ Chong. 3hbac (3-hop between adjacent clusterheads): a novel non-overlapping clustering algorithm for mobile ad hoc networks. In Communications, Computers and signal Processing, 2003. PACRIM. 2003 IEEE Pacific Rim Conference on, volume 1, pages 318-321. IEEE, 2003.

[17] Taek Jin Kwon, Mario Gerla, Vijay K Varma, Melbourne Barton, and $\mathrm{T}$ Russell Hsing. Efficient flooding with passive clustering-an overhead-free selective forward mechanism for ad hoc/sensor networks. Proceedings of the IEEE, 91(8):1210-1220, 2003.

[18] Badreddine Guizani, B'echir Ayeb, and Abderrafiaa Koukam. A stable k-hop clustering algorithm for routing in mobile ad hoc networks. In Wireless Communications and Mobile Computing Conference (IWCMC), 2015 International, pages 659-664. IEEE, 2015. 